\title{
The frozen elephant trunk technique for retrograde acute type A aortic dissection
}

\author{
Kentaro Tamura, MD, ${ }^{\mathrm{a}}$ Naomichi Uchida, MD, ${ }^{\mathrm{b}}$ Akira Katayama, MD, ${ }^{\mathrm{c}}$ Miwa Sutoh, MD, \\ Masatsugu Kuraoka, MD, ${ }^{c}$ and Taijiro Sueda, MD $^{b}$
}

Objective: The operative strategies for retrograde acute type A aortic dissection with a primary intimal tear remain controversial because resection of an intimal tear via a median sternotomy is difficult. We evaluated the frozen elephant trunk technique for treating this type of aortic dissection.

\begin{abstract}
Methods: The frozen elephant trunk technique was used for acute retrograde type A aortic dissection with a primary intimal tear in the descending aorta in 25 consecutive patients ( 16 men, 9 women; median age, 64 years) seen between 1997 and 2011 at the Hiroshima City Asa Hospital. Three patients had Marfan syndrome. Fourteen patients had ischemia of the lower half of the body, 2 had stroke, 3 had coronary ischemia, and 10 had pericardial tamponade.

Results: There were no hospital deaths. Postoperative complications occurred in 2 patients, including stroke and mediastinitis. Two late deaths occurred over a median follow-up period of 58 months (12-169 months), one caused by stroke and the other by colon cancer. Two patients needed additional operations: one required a second operation for descending aorta replacement because of ulceration caused by the stent graft, and the other required an endovascular stent graft because of dilatation of the descending aorta. Computed tomography follow-up scans revealed that the false lumen was completely thrombosed and obliterated in all 25 patients with stent grafts.
\end{abstract}

Conclusions: The frozen elephant trunk technique for retrograde type A aortic dissection yielded acceptable outcomes, despite the high preoperative morbidity, and might improve the prognosis of dissection. (J Thorac Cardiovasc Surg 2014;148:561-5)

Tear-oriented surgery for type A acute aortic dissection has been widely recommended. However, the operative strategies for acute type A aortic dissection with a primary intimal tear in the descending aorta, so-called retrograde type A dissection, remain controversial. Arch replacement combined with antegrade stent grafting into the descending aorta via the aortic arch (frozen elephant trunk [FET]) represents a hybrid surgical approach to cover the primary intimal tear in retrograde type A aortic dissection. This article describes the results of a retrospective evaluation of the effectiveness of the FET technique for the treatment of retrograde acute type A aortic dissection.

\section{PATIENTS AND METHODS Patients}

The clinical records of 25 patients who underwent total arch replacement by the FET technique for type A aortic dissection with a

\footnotetext{
From Division of Cardiovascular Surgery, ${ }^{\mathrm{a}}$ The Sakakibara Heart Institute of

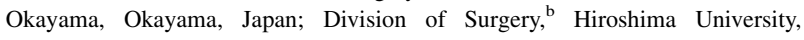
Hiroshima, Japan; and Division of Cardiovascular Surgery, ${ }^{\mathrm{c}}$ Hiroshima City Asa Hospital, Hiroshima, Japan.

Disclosures: Authors have nothing to disclose with regard to commercial support. Received for publication April 25, 2012; revisions received Oct 26, 2012; accepted for publication Dec 5, 2012; available ahead of print Nov 18, 2013

Address for reprints: Kentaro Tamura, MD, Division of Cardiovascular Surgery, The

Sakakibara Heart Institute of Okayama, 2-1-5, Nakaicho, Kita-Ku, Okayama,

700-0804, Japan (E-mail: tamuken0801@yahoo.co.jp).

$0022-5223 / \$ 36.00$

Copyright (c) 2014 by The American Association for Thoracic Surgery

http://dx.doi.org/10.1016/j.jtcvs.2012.12.094
}

primary intimal tear in the descending aorta, so-called retrograde acute type A aortic dissection, between December 1997 and February 2011 were retrospectively reviewed. Twenty-five (17\%) of 150 consecutive patients who had undergone surgery for acute type A aortic dissection at the Hiroshima City Asa Hospital during that period were included in the study. A primary intimal tear was surgically closed using the FET technique in all patients who had retrograde acute type A aortic dissection There were 16 men and 9 women; the median age was 64 years (range, 32-80 years). Three patients had Marfan syndrome. Two patients had stroke, 3 had coronary ischemia, and 10 had pericardial tamponade. Ischemia of the lower half of the body was found by preoperative computed tomography (CT) angiography in 14 patients $(56 \%)$, including 9 with limb ischemia, 8 with renal ischemia, and 7 with mesenteric ischemia (Table 1). The stent grafts were hand-made using 2 Gianturco Z-stents (Cook Inc, Bloomington, Ind), which were preconstructed, and covered with a prosthetic vascular graft ( $\mathrm{J}$ graft, Japan Lifeline Co, Japan). The use of the hand-made stent graft for the FET was approved by the Ethics Committee. The retrospective review of the records for possible publication was also approved by the Institutional Review Board.

\section{Operative Procedures}

The FET technique has been described in our previous reports. ${ }^{1}$ A median sternotomy was performed after induction of general anesthesia An arterial cannula was inserted into the right axillary artery, femoral artery, or both; venous drainage cannulas were inserted into the superior and inferior vena cava and total extracorporeal circulation was commenced. After the rectal temperature decreased to $28^{\circ} \mathrm{C}$, the aortic arch was incised longitudinally until immediately before the origin of the left subclavian artery under lower circulatory arrest with selective cerebral perfusion (Figure 1, $A$ and $B$ ). At the end of this incision, the aortic arch was dissected transversely and a ball-shaped valve sizer was inserted into the true lumen of the descending aorta guided by transesophageal ultrasonography, because it was sometimes difficult to visualize the 


\section{Abbreviations and Acronyms \\ $\mathrm{CT}=$ computed tomography \\ $\mathrm{FET}=$ frozen elephant trunk}

primary intimal tear, to verify the correct size of the FET (diameter and length). A stent graft $5 \%$ to $10 \%$ smaller than the total aortic diameter was chosen intraoperatively to stent the aorta down to the T6 vertebral level 2 to $3 \mathrm{~cm}$ above the level of the aortic valve. The FET was placed through a $30 \mathrm{~F}$ introducer inserted into the descending aorta. The graft was fixed in the true lumen of the descending aorta by expansion of the $\mathrm{Z}$ stent. The left subclavian artery was dissected transversely at the proximal end and the proximal stump was closed. The adventitia of the aortic stump was covered with a felt strip 2-cm wide, and the stump was reinforced with continuous 4-0 polypropylene sutures. A synthetic graft with 4 branches was anastomosed end to end to the stump of the distal aortic arch. The third branch was anastomosed to the left subclavian artery. The proximal graft was crossclamped, antegrade systemic perfusion from the fourth branch was started, and the patient was rewarmed by means of extracorporeal circulation. The left common carotid artery and the brachiocephalic artery were anastomosed to respective branches of the graft. Then the proximal graft was anastomosed to the stump of the ascending aorta to complete the procedure (Figure 1, $A$ ).

Concomitant procedures included 3 cases of coronary artery bypass grafting. The median operative time and times for the cardiopulmonary bypass, selective cerebral perfusion, heart ischemic, and circulatory arrest of the lower body were 330,163, 69, 90 and 34 minutes, respectively. The median diameter of the stent graft was $28 \mathrm{~mm}$, and the median length was $10 \mathrm{~cm}$. The stent graft was delivered to the level of the fifth thoracic vertebra (T5) in 2 patients, T6 in 18 patients, and T7 in 5 patients (Table 2).

\section{Patient Follow-up}

The follow-up protocol included postoperative enhanced CT at 1 month, 1 year, 3 years, and 5 years after the surgery. The median follow-up period was 58 months (12-169 months). A follow-up CT image was available in $100 \%(25 / 25)$ of patients.

\section{RESULTS}

None of the patients died in the hospital (hospital mortality, $0 \%$ ). Perioperative morbidity included renal failure that necessitated transient continuous hemodialysis in 3 patients, respiratory failure in 2 patients, stroke in 1 patient, mediastinitis in 1 patient, and reexploration for bleeding in 1 patient. All patients recovered fully. No patients developed paraplegia or mesenteric necrosis in this series. Of the 25 patients, $24(96 \%)$ were discharged and sent home.

The median follow-up period was 58 months. There were 2 late deaths in this series: caused by colon cancer in one patient 162 months after the operation, and by cerebral infarction in the other at 69 months after the operation. Survival was $87.5 \%$ at 10 years (Figure 2). Two patients without Marfan syndrome needed additional operations. One patient required a second operation for descending aorta replacement at 11 months after the first operation because of dilatation of the descending aorta with a residual tear that could not be covered by a stent graft, and the other patient required endovascular stent grafting in the
TABLE 1. Patient characteristics

\begin{tabular}{lc}
\hline Number of patients & 25 \\
Male & 16 \\
Marfan syndrome & 3 \\
Age, years (median) & $32-80(64)$ \\
Interval from symptom onset to surgery & \\
Within 6 hours & 20 \\
6 hours to 24 hours & 5 \\
Preoperative comorbidity & \\
Stroke & 2 \\
Right radial arterial pulse weakness & 4 \\
Pericardial tamponade & 10 \\
Coronary ischemia & 3 \\
Visceral ischemia & 7 \\
Renal ischemia & 8 \\
Limb ischemia & 9 \\
Renal failure (serum creatinine $>2.0 \mathrm{mg} / \mathrm{dL})$ & 3 \\
\hline
\end{tabular}

descending aorta at 28 months after the first surgery, because of an ulceration-like aneurysm distal to the stent graft (Table 3). Three patients with Marfan syndrome are alive and well with no reinterventions. Freedom from an overall aortic event was $88.9 \%$ at 10 years (Figure 3). On the other hand, the false lumen below the distal descending aorta (T11 level) was patent in 8 patients, including 2 patients with Marfan syndrome, associated with reentry on the abdominal aorta and/or iliac artery. However, no patients showed dilatation in excess of $40 \mathrm{~mm}$ of the thoracoabdominal aorta.

\section{DISCUSSION}

Intimal tears in cases of type A acute aortic dissection are usually located in the ascending aorta, however, in some cases of dissection of the ascending aorta, the intimal tear may be present in the descending aorta. In these cases, the dissection extends not only in an antegrade direction but also in a retrograde direction toward the aortic valve, and may be associated with high mortality. Based on our experience, patients with retrograde dissection of the ascending aorta show a high rate of preoperative comorbidities. Fourteen patients $(56 \%)$ in this study had ischemia of the lower half of the body. However, there have been few reports on acute retrograde dissection of the ascending aorta. Roberts and Roberts ${ }^{2}$ reported on 12 patients who were found to have the entrance tear in the transverse aorta at autopsy; the longitudinal extension of the aortic dissection was only in the retrograde direction in 4 patients $(33 \%)$, only in the antegrade direction in 4 patients $(33 \%)$, and in both directions in 4 patients $(33 \%)$. von Segesser and colleagues ${ }^{3}$ reported on 9 $(10 \%)$ of 89 consecutive patients with type A dissection who were surgically treated for an intimal tear in the descending aorta. Hanafusa and colleagues ${ }^{4}$ reported that $12(8 \%)$ of 139 patients were treated surgically. In our 

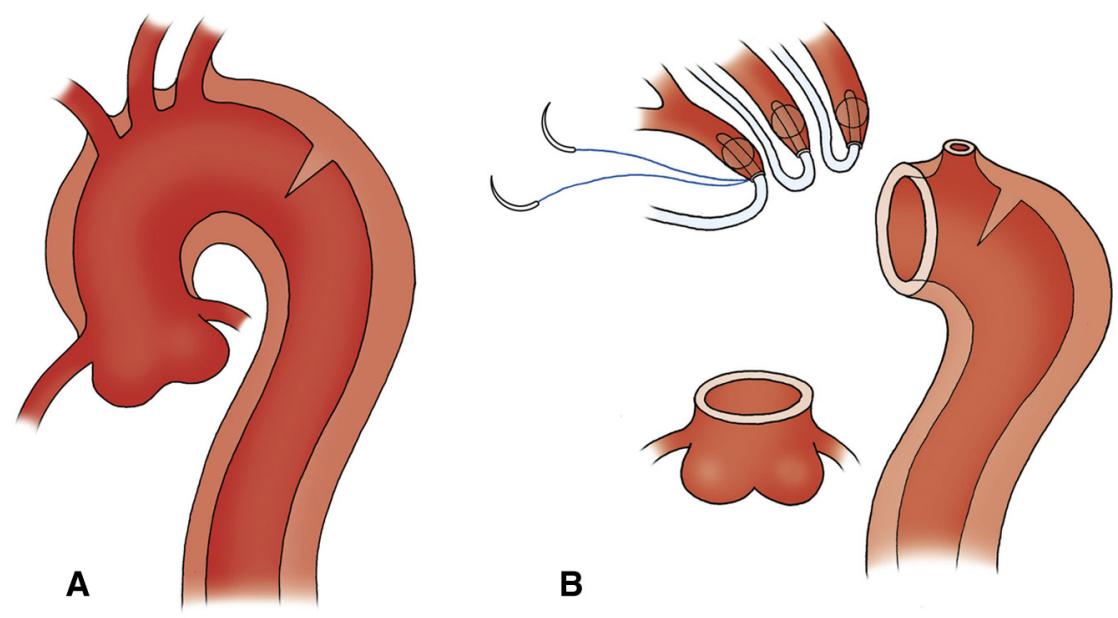

B

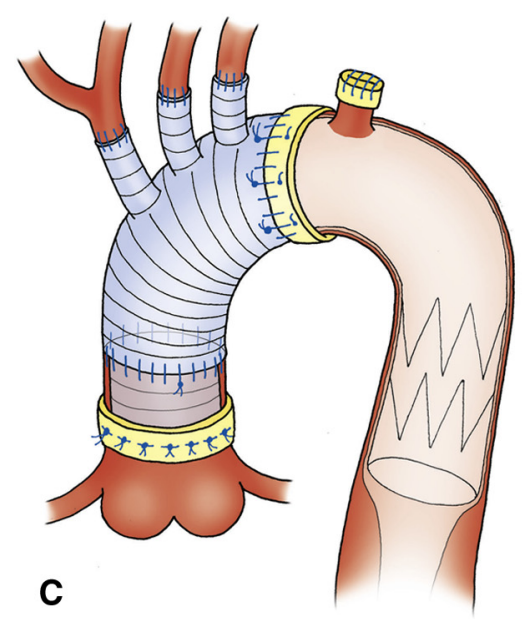

FIGURE 1. Retrograde type A aortic dissection with primary intimal tear in the descending aorta (A). The aortic arch was incised longitudinally until immediately before the origin of the left subclavian artery under lower circulatory arrest with selective cerebral perfusion (B). After the frozen elephant trunk was inserted into the descending aorta, the adventitia of the aortic stump was reinforced with a felt strip. A synthetic graft with 4 branches was anastomosed to the aortic stump (C).

series, the FET technique was applied in $25(17 \%)$ of 150 consecutive patients with acute type A dissection.

The elephant trunk technique has been widely used for acute type A aortic dissection. ${ }^{5-7}$ Kazui and colleagues ${ }^{7}$ performed aortic arch repair for acute type A dissection with an intimal tear in the aortic arch or descending aorta to prevent dilation of the false lumen in the aortic arch in the late postoperative period. However, the diameter of the graft inserted using the elephant trunk procedure is likely to be smaller than that of a distally stented graft. Use of a distally anchored stent graft allows insertion of a prosthesis with a larger diameter and longer length than the elephant trunk method. When grafting is undertaken using the elephant trunk procedure, the graft inserted is often smaller than that required in relation to the diameter of the true lumen, and therefore, dilation of the residual false lumen cannot be entirely prevented. The aim of this hybrid FET technique is not only to repair intimal tears in the proximal descending aorta, the so-called retrograde type A aortic dissection using a stent graft, but also to exclude all antegrade blood flow in the false lumen of the thoracic aorta while more fully reexpanding the true lumen and obliterating the false lumen in the descending aorta; this may provide improved restoration of distal visceral and limb perfusion, and prevent aortic dilatation after surgery. Hoffman and colleagues ${ }^{8}$ reported that at midterm follow-up, choosing a stent the size of the total aortic diameter (true and false lumen) allows immediate closure of the false lumen of the aortic dissection, ensuring obliteration of all intercostal arteries without any signs of endoleak or residual patency of the false lumen. We determined the most appropriate size of the stent graft by measuring the diameter of the true lumen with a ball-shaped sizer during systemic circulatory arrest under transesophageal echocardiographic guidance. In all patients with and without Marfan syndrome, a stent graft $5 \%$ to 
TABLE 2. Operative variables

\begin{tabular}{lr} 
Median surgical time, min & 330 \\
Median cardiopulmonary bypass time, min & 163 \\
Median selective cerebral perfusion time, min & 69 \\
Median heart ischemic time, min & 90 \\
Median time of circulatory arrest of the lower body, min & 34 \\
Arterial perfusion site & 22 \\
Unilateral femoral artery & 10 \\
Both femoral arteries & 3 \\
Right axillary artery & 7 \\
Left ventricular apex & 2 \\
Stent graft for frozen elephant trunk & \\
Median diameter, mm & 28 \\
Median length, cm & 10 \\
Distal landing zone & \\
T5 & 2 \\
T6 & 18 \\
T7 & 5 \\
Coronary artery bypass grafting & 3 \\
Right coronary artery & \\
\hline
\end{tabular}

$10 \%$ smaller than the total aortic diameter was chosen intraoperatively to stent the aorta down to the T5 to T7 vertebral level 2 to $3 \mathrm{~cm}$ above the level of the aortic valve.

It has been reported that the FET technique is less invasive and yields good midterm results. ${ }^{1,9-11}$ In the case of retrograde dissection with an intimal tear located in the descending aorta, where it cannot be reached via a median sternotomy, use of the FET technique can allow closure of the tear, with avoidance of a left thoracotomy. In addition, the kinking and flapping action of the elephant trunk can be prevented and the FET allows progressive thrombus formation in the perigraft space in the descending aorta up to the level of the stent. In the present study, all patients showed thrombosis of the false lumen around the FET, which may be well correlated with reverse remodeling of the aorta, with consequent reduction in the aortic diameter and volume.

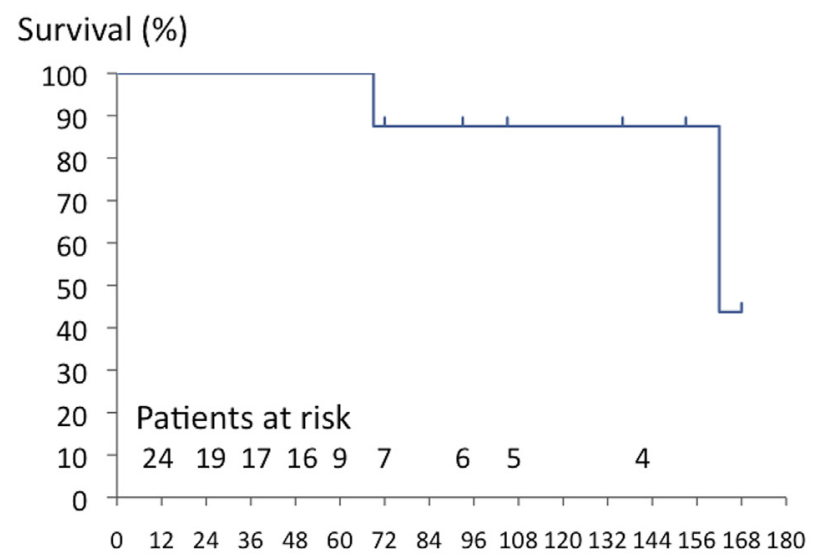

(Months)

FIGURE 2. The 10-year survival was $87.5 \%$.
TABLE 3. Postoperative variables

\begin{tabular}{lc}
\hline Early mortality & \\
Operative death & 0 \\
In-hospital death & \\
Early morbidities & 1 \\
$\quad$ Stroke & 0 \\
Spinal cord injury & 3 \\
Renal failure (transient) & 1 \\
Reexploration for bleeding & 1 \\
Mediastinitis & 0 \\
Intestinal necrosis & $12-169(58)$ \\
Follow-up period, mo (median) & 2 \\
Late mortality & 1 \\
$\quad$ Stroke & 1 \\
Cancer & 2 \\
Late morbidities & \\
Additional operation* & 0 \\
CT scan (n = 25) $\dagger$ & \\
\hline$C T$, Computed tomography. *One endovascular stent graft for the descending aorta \\
and 1 replacement of the descending aorta. $\dagger$ Patent false lumen on the stent graft.
\end{tabular}

In this study, 3 patients with Marfan syndrome successfully underwent the FET technique. Sun and colleagues ${ }^{12}$ treated 19 patients with Marfan syndrome with this procedure and hospital mortality was $0 \%$. But the distal end of the stent graft was seen to enter the false lumen in 4 patients $(21.1 \%)$. Careful manipulation is required when using the FET technique because of the fragility and weakness of the aortic wall. The prognosis for patients with Marfan syndrome with the FET technique is still unknown and close long-term follow-up is required to evaluate the effectiveness of this procedure.

Spinal cord ischemia is the most serious complication of the FET technique. ${ }^{1,13-15}$ Karck and Kamiya ${ }^{15}$ reviewed the use of FET in 215 patients from 8 reports, and the incidence of paraplegia after implantation of the FET was 3.3\%. According to a recent report, however, this usually occurs when a stent graft is used for an arteriosclerotic aneurysm with debris or a chronic aortic dissection without

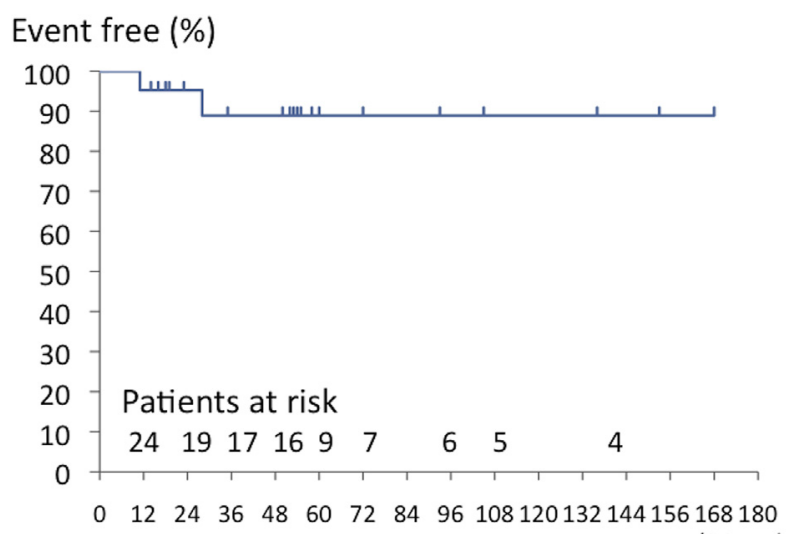

(Months)

FIGURE 3. Aortic events. The 10 -year event-free rate was $88.9 \%$. 
communication between the true and false lumen. ${ }^{16}$ In our series, the mean length of the stent graft was $10.2 \mathrm{~cm}$ and the FET was inserted as far as the T7 level to prevent the vital intercostal arteries from being occluded by the FET, and no paraplegia occurred.

\section{Limitations}

The study is too small to conclude that the FET technique is more effective for distal remodeling than the conventional elephant trunk procedure. This study was retrospectively reviewed. Further randomization with comparison of each technique and endovascular stent grafting is required to provide precise conclusions about the ideal procedure to treat retrograde aortic dissection.

\section{CONCLUSIONS}

The FET technique for retrograde acute type A aortic dissection has an acceptable outcome despite high preoperative morbidity and might improve the prognosis of dissection.

\section{References}

1. Uchida N, Ishihara H, Shibamura H, Kyo Y, Ozawa M. Midterm results of extensive primary repair of the thoracic aorta by means of total arch replacement with open stent graft replacement for an acute type A aortic dissection. J Thorac Cardiovasc Surg. 2006;131:862-7.

2. Roberts CS, Roberts WC. Aortic dissection with the entrance tear in transverse aorta: analysis of 12 autopsy patients. Ann Thorac Surg. 1990;50:762-6.

3. von Segesser LK, Killer I, Ziswiler M, Linka A, Ritter M, Jenni R, et al. Dissection of the descending thoracic aorta extending into the ascending aorta. J Thorac Cardiovasc Surg. 1994;108:755-61.

4. Hanafusa Y, Ogino H, Sasaki H, Minatoya K, Ando M, Okita Y, et al. Total arch replacement with elephant trunk procedure for retrograde dissection. Ann Thorac Surg. 2002;74:S1836-9.
5. Crawford ES, Coseli JS, Svensson LG, Safi HJ, Hess KR. Diffuse aneurysmal disease (chronic aortic dissection, Marfan, and mega aorta syndromes) and multiple aneurysm: treatment by subtotal and total aortic replacement emphasizing the elephant trunk operation. Ann Surg. 1990;211:521-37.

6. Heinemeann MK, Buehner B, Jurmann MJ, Borst HG. Use of the "Elephant trunk technique" in aortic surgery. Ann Thorac Surg. 1995;60:2-6.

7. Kazui T, Washiyama N, Muhammad BA, Terada H, Yamashita K, Takinami M, et al. Extended total arch replacement for acute type A aortic dissection: experience with seventy patients. J Thorac Cardiovasc Surg. 2000;119:558-65

8. Hoffman A, Damberg AL, Schälte G, Mahnken AH, Raweh A, Autschbach R. Thoracic stent graft sizing for frozen elephant trunk repair in acute type A dissection. J Thorac Cardiovasc Surg. 2013;145:964-9.

9. Kato M, Kuratani T, Kaneko M, Kyo S, Ohnishi K. The results of total arch graft implantation with open stent-graft placement for type A aortic dissection. J Thorac Cardiovasc Surg. 2002;124:531-40.

10. Di Bartolomeo R, Pacini D, Savini C, Pilato E, Martin-Suarez S, Di Marco L, et al. Complex thoracic aortic disease: single-stage procedure with the frozen elephant trunk technique. J Thorac Cardiovasc Surg. 2010;140:S81-5.

11. Liu ZG, Sun LZ, Chang Q, Zhu JM, Dong C, Yu CT, et al. Should the "elephant trunk" be skeletonized? Total arch replacement combined with stented elephan trunk implantation for Stanford A type A aortic dissection. J Thorac Cardiovasc Surg. 2006;131:107-13.

12. Sun LZ, Li M, Zhu JM, Liu YM, Chang Q, Zheng J, et al. Surgery for patients with Marfan syndrome with type A dissection involving the aortic arch using total arch replacement combined with stented elephant trunk implantation: the acute versus the chronic. J Thorac Cardiovasc Surg. 2011 142:e85-91.

13. Flores J, Kunihara T, Shiiya N, Yoshimoto K, Matsuzaki K, Yasuda K. Extensive deployment of the stented elephant trunk is associated with an increased risk of spinal cord injury. J Thorac Cardiovasc Surg. 2006;131:336-42.

14. Usui A, Fujimoto K, Ishiguthi T, Yoshikawa M, Akita T, Ueda Y. Cerebrospinal dysfunction after endovascular stent grafting via a median sternotomy: the frozen elephant trunk procedure. Ann Thorac Surg. 2002;74:1821-4.

15. Karck M, Kamiya H. Progress of the treatment for extended aortic aneurysms: is the frozen elephant trunk technique the next standard in the treatment of complex aortic disease including arch? Eur J Cardiothorac Surg. 2008;33: $1007-13$.

16. Ius F, Hagl C, Harverich A, Pichlmaier M. Elephant trunk procedure 27 years af ter Boast: what remains and what is new? Eur J Cardiothorac Surg. 2011;40: $1-12$. 